\title{
A generalization of Čbyšev type inequalities for first differentiable mappings
}

Erhan Set, Mehmet Zeki Sarikaya, and Farooq Ahmad 


\title{
A GENERALIZATION OF ČEBYŠEV TYPE INEQUALITIES FOR FIRST DIFFERENTIABLE MAPPINGS
}

\author{
ERHAN SET, MEHMET ZEKI SARIKAYA, AND FAROOQ AHMAD
}

Received February 16, 2011

\begin{abstract}
In this paper, we improve and further generalize some Čebyšev type inequalities involving functions whose derivatives belong to $L_{p}$ spaces via certain integral identities.

2000 Mathematics Subject Classification: 26D07, 26D15, 26D20

Keywords: Čebyšev type inequalities, $L_{p}$ spaces
\end{abstract}

\section{INTRODUCTION}

In 1882, P. L. Čebyšev proved the following important integral inequality [13],[9, p.207]:

$$
T(f, g) \leq \frac{1}{12}(b-a)^{2}\left\|f^{\prime}\right\|_{\infty}\left\|g^{\prime}\right\|_{\infty},
$$

where $f, g:[a, b] \rightarrow \mathbb{R}$ are absolutely continuous functions whose derivatives $f^{\prime}, g^{\prime} \in$ $L_{\infty}[a, b]$ and

$$
T(f, g)=\frac{1}{b-a} \int_{a}^{b} f(x) g(x) d x-\left(\frac{1}{b-a} \int_{a}^{b} f(x) d x\right)\left(\frac{1}{b-a} \int_{a}^{b} g(x) d x\right),
$$

which is called the Čebyšev functional, provided the integrals in (1.2) exist.

During the past few years many researchers have given considerable attention to the inequality of (1.1). Various generalizations, extensions and variants of this inequality have appeared in the literature, see ([1-13]) and the references cited therein.

Recently, Pachpatte [8] established a new Čebyšev type inequality involving functions whose derivatives belong to $L_{p}$ spaces:

Theorem 1. Let $f, g:[a, b] \rightarrow \mathbb{R}$ are absolutely continuous functions whose derivatives $f^{\prime}, g^{\prime} \in L_{p}[a, b], p>1$. Then, we have inequalities

$$
T(f, g) \leq \frac{1}{(b-a)^{3}}\left\|f^{\prime}\right\|_{p}\left\|g^{\prime}\right\|_{p} \int_{a}^{b}(B(x))^{\frac{2}{q}} d x,
$$


and

$$
T(f, g) \leq \frac{1}{2(b-a)^{2}} \int_{a}^{b}\left[g(x)\left\|f^{\prime}\right\|_{p}+f(x)\left\|g^{\prime}\right\|_{p}\right](B(x))^{\frac{1}{q}} d x,
$$

where

$$
B(x)=\frac{(x-a)^{q+1}+(b-x)^{q+1}}{q+1},
$$

for $x \in[a, b]$ and $\frac{1}{p}+\frac{1}{q}=1$.

Motivated by the results of Pachpatte, in the present paper we establish some new Čebyšev type inequalities for $\frac{1}{p}+\frac{1}{q}=1$. The analysis used in the proofs is elementary and our results provide new estimates for these types of inequalities.

\section{MAIN RESULTS}

Let $[a, b] \subset \mathbb{R}, a<b$; and as usual for any function $h \in L_{p}[a, b], p>1$, we define $\|h\|_{p}=\left(\int_{a}^{b}|h(t)|^{p} d t\right)^{\frac{1}{p}}$. We use the following notation to simplify the details of presentation. For suitable functions $f, g:[a, b] \rightarrow \mathbb{R}$ and $\lambda \in[0,1]$, we set

$$
\begin{aligned}
& T_{\lambda}(f, g) \\
= & \frac{1}{b-a}\left(1-\frac{\lambda}{2}\right) \int_{a}^{b} f(x) g(x) d x \\
& -\frac{\lambda}{4(b-a)^{2}}\left\{\int_{a}^{b}[(x-b) f(b)-(x-a) f(a)] g(x) d x\right. \\
& \left.\quad+\int_{a}^{b}[(x-b) g(b)-(x-a) g(a)] f(x) d x\right\} \\
& -\left(\frac{1}{b-a} \int_{a}^{b} f(x) d x\right)\left(\frac{1}{b-a} \int_{a}^{b} g(x) d x\right) .
\end{aligned}
$$

Now, let us state our main results.

Theorem 2. Let $f, g:[a, b] \rightarrow \mathbb{R}$ be absolutely continuous functions whose derivatives $f^{\prime}, g^{\prime} \in L_{p}[a, b], p>1$. Then, the following inequality holds:

$$
\left|T_{\lambda}(f, g)\right| \leq \frac{1}{2(b-a)^{2}} \int_{a}^{b}\left[g(x)\left\|f^{\prime}\right\|_{p}+f(x)\left\|g^{\prime}\right\|_{p}\right](Z(x))^{\frac{1}{q}} d x,
$$

where

$$
Z(x)=\frac{(x-a)^{q+1}+(b-x)^{q+1}}{q+1}\left(\left(\frac{\lambda}{2}\right)^{q+1}+\left(1-\frac{\lambda}{2}\right)^{q+1}\right),
$$

for $x \in[a, b], \frac{1}{p}+\frac{1}{q}=1$ and $\lambda \in[0,1]$. 
Proof. From the hypotheses, we can write the following identities,

$$
\begin{gathered}
\frac{1}{b-a} \int_{a}^{b} p(x, t) f^{\prime}(t) d t \\
=\left(1-\frac{\lambda}{2}\right) f(x)-\lambda \frac{(x-b) f(b)-(x-a) f(a)}{2(b-a)}-\frac{1}{b-a} \int_{a}^{b} f(t) d t,
\end{gathered}
$$

and

$$
\begin{gathered}
\frac{1}{b-a} \int_{a}^{b} p(x, t) g^{\prime}(t) d t \\
=\left(1-\frac{\lambda}{2}\right) g(x)-\lambda \frac{(x-b) g(b)-(x-a) g(a)}{2(b-a)}-\frac{1}{b-a} \int_{a}^{b} g(t) d t,
\end{gathered}
$$

for $x \in[a, b], \lambda \in[0,1]$, where

$$
p(x, t)=\left\{\begin{array}{ll}
t-\left(a+\lambda \frac{x-a}{2}\right), & a \leq t \leq x \\
t-\left(b-\lambda \frac{b-x}{2}\right), & x<t \leq b
\end{array} .\right.
$$

A simple proof of equalities of (2.2) and (2.3) can be done by integrating in the left hand side and using the identity of $p(x, t)$. The details are left for the interested reader.

Multiplying both sides of (2.2) and (2.3) by $g(x)$ and $f(x)$ respectively and adding the resulting identities, we get

$$
\begin{aligned}
& \frac{1}{b-a} g(x) \int_{a}^{b} p(x, t) f^{\prime}(t) d t+\frac{1}{b-a} f(x) \int_{a}^{b} p(x, t) g^{\prime}(t) d t \\
= & 2\left(1-\frac{\lambda}{2}\right) f(x) g(x)-\lambda \frac{(x-b) f(b)-(x-a) f(a)}{2(b-a)} g(x) \\
& -\lambda \frac{(x-b) g(b)-(x-a) g(a)}{2(b-a)} f(x) \\
& -\frac{1}{b-a} g(x) \int_{a}^{b} f(t) d t-\frac{1}{b-a} f(x) \int_{a}^{b} g(t) d t .
\end{aligned}
$$

Multiplying both sides of (2.4) by $\frac{1}{b-a}$, we get

$$
\begin{aligned}
& \frac{2}{b-a}\left(1-\frac{\lambda}{2}\right) f(x) g(x) \\
& -\lambda \frac{(x-b) f(b)-(x-a) f(a)}{2(b-a)^{2}} g(x)-\lambda \frac{(x-b) g(b)-(x-a) g(a)}{2(b-a)^{2}} f(x) \\
& -\frac{1}{(b-a)^{2}} g(x) \int_{a}^{b} f(t) d t-\frac{1}{(b-a)^{2}} f(x) \int_{a}^{b} g(t) d t
\end{aligned}
$$




$$
=\frac{1}{(b-a)^{2}} g(x) \int_{a}^{b} p(x, t) f^{\prime}(t) d t+\frac{1}{(b-a)^{2}} f(x) \int_{a}^{b} p(x, t) g^{\prime}(t) d t .
$$

Integrating both sides of (2.5) with respect to $x$ over $[a, b]$, we get

$$
\begin{aligned}
& \quad \frac{2}{b-a}\left(1-\frac{\lambda}{2}\right) \int_{a}^{b} f(x) g(x) d x \\
& -\frac{\lambda}{2(b-a)^{2}}\left\{\int_{a}^{b}[(x-b) f(b)-(x-a) f(a)] g(x) d x\right. \\
& \left.+\int_{a}^{b}[(x-b) g(b)-(x-a) g(a)] f(x) d x\right\} \\
& -2\left(\frac{1}{b-a} \int_{a}^{b} g(x) d x\right)\left(\frac{1}{b-a} \int_{a}^{b} f(x) d x\right) \\
& =\frac{1}{(b-a)^{2}} \int_{a}^{b} g(x)\left(\int_{a}^{b} p(x, t) f^{\prime}(t) d t\right) d x \\
& +\frac{1}{(b-a)^{2}} \int_{a}^{b} f(x)\left(\int_{a}^{b} p(x, t) g^{\prime}(t) d t\right) d x .
\end{aligned}
$$

After the rewriting of the equality of (2.5), we obtain

$$
T_{\lambda}(f, g)=\frac{1}{2(b-a)^{2}} \int_{a}^{b}\left[g(x) \int_{a}^{b} p(x, t) f^{\prime}(t) d t+f(x) \int_{a}^{b} p(x, t) g^{\prime}(t) d t\right] d x \text {. }
$$

From (2.7) and using the properties of modulus, Hölder's integral inequality, we have

$$
\begin{gathered}
\left|T_{\lambda}(f, g)\right| \\
\leq \frac{1}{2(b-a)^{2}} \int_{a}^{b}\left[|g(x)| \int_{a}^{b}|p(x, t)|\left|f^{\prime}(t)\right| d t+|f(x)| \int_{a}^{b}|p(x, t)|\left|g^{\prime}(t)\right| d t\right] d x \\
\leq \frac{1}{2(b-a)^{2}} \int_{a}^{b}\left[|g(x)|\left(\int_{a}^{b}|p(x, t)|^{q} d t\right)^{\frac{1}{q}}\left(\int_{a}^{b}\left|f^{\prime}(t)\right|^{p} d t\right)^{\frac{1}{p}}\right. \\
\left.+|f(x)|\left(\int_{a}^{b}|p(x, t)|^{q} d t\right)^{\frac{1}{q}}\left(\int_{a}^{b}\left|g^{\prime}(t)\right|^{p} d t\right)^{\frac{1}{p}}\right] d x \\
=\frac{1}{2(b-a)^{2}} \int_{a}^{b}\left[g(x)\left\|f^{\prime}\right\|_{p}+f(x)\left\|g^{\prime}\right\|_{p}\right]\left(\int_{a}^{b}|p(x, t)|^{q} d t\right)^{\frac{1}{q}} d x
\end{gathered}
$$


A simple calculation shows that

$$
\begin{aligned}
& \int_{a}^{b}|p(x, t)|^{q} d t \\
= & \int_{a}^{x}\left|t-\left(a+\lambda \frac{x-a}{2}\right)\right|^{q} d t+\int_{x}^{b}\left|t-\left(b-\lambda \frac{b-x}{2}\right)\right|^{q} d t \\
= & (x-a)^{q} \int_{a}^{x}\left|\frac{t-a}{x-a}-\frac{\lambda}{2}\right|^{q} d t+(b-x)^{q} \int_{x}^{b}\left|\frac{t-b}{b-x}-\frac{\lambda}{2}\right|^{q} d t \\
= & \frac{(x-a)^{q+1}+(b-x)^{q+1}}{q+1}\left(\left(\frac{\lambda}{2}\right)^{q+1}+\left(1-\frac{\lambda}{2}\right)^{q+1}\right) \\
= & Z(x) .
\end{aligned}
$$

Using (2.8) and (2.9), we get the required inequality in (2.1). The proof is complete.

Remark 1. If we choose $\lambda=0$ in (2.1), then we get (1.3).

Corollary 1. Let $f:[a, b] \rightarrow \mathbb{R}$ be an absolutely continuous function whose derivative $f^{\prime} \in L_{p}[a, b], p>1$. Then, the following inequality holds:

$$
\begin{gathered}
\left|\left(1-\frac{\lambda}{2}\right) f(x)-\lambda \frac{(x-b) f(b)-(x-a) f(a)}{2(b-a)}-\frac{1}{b-a} \int_{a}^{b} f(t) d t\right| \\
\leq \frac{1}{b-a}\left\|f^{\prime}\right\|_{p}(Z(x))^{\frac{1}{q}} .
\end{gathered}
$$

Proof. From (2.2) using the properties of modulus and applying Hölder's integral inequalty, we have

$$
\begin{aligned}
& \left|\left(1-\frac{\lambda}{2}\right) f(x)-\lambda \frac{(x-b) f(b)-(x-a) f(a)}{2(b-a)}-\frac{1}{b-a} \int_{a}^{b} f(t) d t\right| \\
& \quad \leq \frac{1}{b-a}\left(\int_{a}^{b}\left|f^{\prime}(t)\right|^{p} d t\right)^{\frac{1}{p}}\left(\int_{a}^{b}|p(x, t)|^{q} d t\right)^{\frac{1}{q}} \\
& \quad=\frac{1}{b-a}\left\|f^{\prime}\right\|_{p}(Z(x))^{\frac{1}{q}} .
\end{aligned}
$$

Remark 2. i) If we choose $\lambda=1$ in (2.10), then we get

$$
\left|\frac{1}{2} f(x)-\frac{(x-b) f(b)-(x-a) f(a)}{2(b-a)}-\frac{1}{b-a} \int_{a}^{b} f(t) d t\right|
$$




$$
\leq \frac{1}{2(b-a)}\left(\frac{(x-a)^{q+1}+(b-x)^{q+1}}{(q+1)}\right)^{\frac{1}{q}}\left\|f^{\prime}\right\|_{p} .
$$

ii) For $\lambda=0$ in (2.10), we have,

$$
\left|f(x)-\frac{1}{b-a} \int_{a}^{b} f(t) d t\right| \leq \frac{1}{(b-a)}\left(\frac{(x-a)^{q+1}+(b-x)^{q+1}}{(q+1)}\right)^{\frac{1}{q}}\left\|f^{\prime}\right\|_{p} .
$$

Theorem 3. Let $f, g:[a, b] \rightarrow \mathbb{R}$ be absolutely continuous functions whose derivatives $f^{\prime}, g^{\prime} \in L[a, b]$. If $\left|f^{\prime}\right|^{q}$ and $\left|g^{\prime}\right|^{q}$ is convex on $[a, b], p>1, \frac{1}{p}+\frac{1}{q}=1$. Then, the following inequality holds:

$$
\begin{aligned}
& |T(f, g)| \\
& \leq \frac{1}{2} \int_{a}^{b}\left\{\left[|f(x)|\left(\frac{\left|g^{\prime}(a)\right|^{q}+\left|g^{\prime}(b)\right|^{q}}{2}\right)^{\frac{1}{q}}\right.\right. \\
& \left.\left.\quad+|g(x)|\left(\frac{\left|f^{\prime}(a)\right|^{q}+\left|f^{\prime}(b)\right|^{q}}{2}\right)^{\frac{1}{q}}\right] M^{\frac{1}{p}}(x)\right\} d x
\end{aligned}
$$

where

$$
M(x)=\frac{(b-x)^{p+1}+(x-a)^{p+1}}{(b-a)^{p+1}(p+1)} .
$$

Proof. From the hypotheses, we can write the following identities,

$$
f(x)=\frac{1}{b-a} \int_{a}^{b} f(x) d x+(b-a) \int_{0}^{1} P(x, t) f^{\prime}(t a+(1-t) b) d t,
$$

and

$$
g(x)=\frac{1}{b-a} \int_{a}^{b} g(x) d x+(b-a) \int_{0}^{1} P(x, t) g^{\prime}(t a+(1-t) b) d t,
$$

for $x \in[a, b]$, where

$$
P(x, t)=\left\{\begin{array}{ll}
t, & 0 \leq t \leq \frac{b-x}{b-a} \\
t-1, & \frac{b-x}{b-a}<t \leq 1
\end{array} .\right.
$$

A simple proof of equalities of (2.12) and (2.13) can be obtained by integrating in the right hand side and using the identity of $p(x, t)$. The details are left for the interested reader.

Multiplying both sides of (2.12) and (2.13) by $g(x)$ and $f(x)$ respectively and adding the resulting identities, we get

$$
2 f(x) g(x)
$$




$$
\begin{aligned}
= & \frac{g(x)}{b-a} \int_{a}^{b} f(x) d x+(b-a) g(x) \int_{0}^{1} P(x, t) f^{\prime}(t a+(1-t) b) d t \\
& +\frac{f(x)}{b-a} \int_{a}^{b} g(x) d x+(b-a) f(x) \int_{0}^{1} P(x, t) g^{\prime}(t a+(1-t) b) d t .
\end{aligned}
$$

Integrating both sides of (2.14) with respect to $x$ over $[a, b]$ and dividing both sides of the resulting identity $(b-a)$, we get

$$
\begin{aligned}
& \frac{2}{b-a} \int_{a}^{b} f(x) g(x) d x \\
= & 2\left(\frac{1}{b-a} \int_{a}^{b} g(x) d x\right)\left(\frac{1}{b-a} \int_{a}^{b} f(x) d x\right) \\
& +\int_{a}^{b} g(x)\left[\int_{0}^{1} P(x, t) f^{\prime}(t a+(1-t) b) d t\right] d x \\
& +\int_{a}^{b} f(x)\left[\int_{0}^{1} P(x, t) g^{\prime}(t a+(1-t) b) d t\right] d x .
\end{aligned}
$$

Rewriting the identity (2.15), we get

$$
\begin{aligned}
T(f, g)= & \frac{1}{2} \int_{a}^{b} g(x)\left[\int_{0}^{1} P(x, t) f^{\prime}(t a+(1-t) b) d t\right] d x \\
& +\frac{1}{2} \int_{a}^{b} f(x)\left[\int_{0}^{1} P(x, t) g^{\prime}(t a+(1-t) b) d t\right] d x .
\end{aligned}
$$

From (2.16), using the properties of modulus and rearranging the terms, we get

$$
\begin{aligned}
& |T(f, g)| \\
\leq & \frac{1}{2} \int_{a}^{b}|f(x)|\left[\int_{0}^{1}|P(x, t)|\left|g^{\prime}(t a+(1-t) b)\right| d t\right] d x \\
& +\frac{1}{2} \int_{a}^{b}|g(x)|\left[\int_{0}^{1}|P(x, t)|\left|f^{\prime}(t a+(1-t) b)\right| d t\right] d x .
\end{aligned}
$$

Applying Hölder's integral inequality and the convexity of $\left|f^{\prime}\right|^{q}$ and $\left|g^{\prime}\right|^{q}$, we obtain

$$
\begin{aligned}
|T(f, g)| \leq & \frac{1}{2} \int_{a}^{b}\left[|f(x)|\left(\int_{0}^{1}|P(x, t)|^{p} d t\right)^{\frac{1}{p}}\left(\int_{0}^{1}\left|g^{\prime}(t a+(1-t) b)\right|^{q} d t\right)^{\frac{1}{q}}\right. \\
& \left.+|g(x)|\left(\int_{0}^{1}|P(x, t)|^{p} d t\right)^{\frac{1}{p}}\left(\int_{0}^{1}\left|f^{\prime}(t a+(1-t) b)\right|^{q} d t\right)^{\frac{1}{q}}\right] d x
\end{aligned}
$$




$$
\begin{aligned}
=\frac{1}{2} \int_{a}^{b}\left\{\left[|f(x)|\left(\frac{\left|g^{\prime}(a)\right|^{q}+\left|g^{\prime}(b)\right|^{q}}{2}\right)^{\frac{1}{q}}\right.\right. \\
\left.\left.+|g(x)|\left(\frac{\left|f^{\prime}(a)\right|^{q}+\left|f^{\prime}(b)\right|^{q}}{2}\right)^{\frac{1}{q}}\right]\left(\int_{0}^{1}|P(x, t)|^{p} d t\right)^{\frac{1}{p}}\right\} d x .
\end{aligned}
$$

A simple computation gives

$$
\begin{aligned}
\int_{0}^{1}|P(x, t)|^{p} d t & =\int_{0}^{\frac{b-x}{b-a}} t^{p} d t+\int_{\frac{b-x}{b-a}}^{1}(t-1)^{p} d t \\
& =\frac{(b-x)^{p+1}+(x-a)^{p+1}}{(b-a)^{p+1}(p+1)}=M(x) .
\end{aligned}
$$

Using (2.18) in (2.18), we get the required inequality in (2.11).

Corollary 2. If in theorem 3 we require that $\left|f^{\prime}(x)\right| \leq K, K>0$ and $\left|g^{\prime}(x)\right| \leq N$, $N>0$, then the following inequality holds

$$
|T(f, g)| \leq \frac{1}{2}\left(\int_{a}^{b}[|f(x)| N+|g(x)| K] M^{\frac{1}{p}}(x) d x\right),
$$

where $\frac{1}{p}+\frac{1}{q}=1$.

\section{REFERENCES}

[1] F. Ahmad, N. S. Barnett, and S. S. Dragomir, "New weighted Ostrowski and Čebyšev type inequalities," Nonlinear Anal., vol. 71, no. 12, pp. e1408-e1412, 2009.

[2] F. Ahmad, P. Cerone, S. S. Dragomir, and N. A. Mir, "On some bounds of Ostrowski and Čebyšev type," J. Math. Inequal., vol. 4, no. 1, pp. 53-65, 2010.

[3] F. Ahmad, A. Rafiq, and N. A. Mir, "Weighted Ostrowski-Grüss type inequality for differentiable mappings," Glob. J. Pure Appl. Math., vol. 2, no. 2, pp. 147-154, 2006.

[4] H. P. Heinig and L. Maligranda, "Chebyshev inequality in function spaces," Real Anal. Exchange, vol. 17, no. 1, pp. 211-247, 1991/92.

[5] D. S. Mitrinović, J. E. Pečarić, and A. M. Fink, Classical and new inequalities in analysis, ser. Math. Appl. (East European Ser.). Dordrecht: Kluwer Academic Publishers Group, 1993, vol. 61.

[6] B. G. Pachpatte, "On trapezoid and Grüss-like integral inequalities," Tamkang J. Math., vol. 34, no. 4, pp. 365-369, 2003.

[7] B. G. Pachpatte, "On Ostrowski-Grüss-Čebyšev type inequalities for functions whose modulus of derivatives are convex," JIPAM. J. Inequal. Pure Appl. Math., vol. 6, no. 4, p. 15, 2005.

[8] B. G. Pachpatte, "On Chebyshev type inequalities involving functions whose derivatives belong to $l_{p}$ spaces," JIPAM. J. Inequal. Pure Appl. Math., vol. 7, no. 2, pp. Article 58, 6, 2006.

[9] J. E. Pečarić, F. Proschan, and Y. L. Tong, Convex functions, partial orderings, and statistical applications, ser. Math. Sci. Engrg. Boston, MA: Academic Press, Inc., 1992, vol. 187.

[10] A. Rafiq and F. Ahmad, "Another weighted Ostrowski-Grüss type inequality for twice differentiable mappings," Kragujevac J. Math., vol. 31, pp. 43-51, 2008. 
[11] A. Rafiq, N. A. Mir, and F. Ahmad, "Weighted Čebyšev-Ostrowski type inequalities," Appl. Math. Mech. (English Ed.), vol. 28, no. 7, pp. 901-906, 2007.

[12] M. Z. Sarikaya, A. Saglam, and H. Yildirim, "On generalization of Čebyšev type inequalities," Iran. J. Math. Sci. Inform., vol. 5, no. 1, pp. 41-48, 2010.

[13] P. L. Čebyšev, "Sur les expressions approximatives des integrales par les auters prises entre les memes limites," Proc. Math. Soc. Charkov, vol. 2, pp. 93-98, 1882.

Authors' addresses

Erhan Set

Department of Mathematics, Faculty of Science and Arts, Düzce University, Düzce-TURKEY

E-mail address: erhanset@yahoo.com

Mehmet Zeki Sarikaya

Department of Mathematics, Faculty of Science and Arts, Düzce University, Düzce-TURKEY

E-mail address: sarikayamz@gmail.com

Farooq Ahmad

Center for Advanced Studies in Pure and Applied Mathematics, B. Z. U., Multan, Pakistan (Presently at Mathematics Department, Govt. Post Graduate College Bhakkar 30000, Punjab, Pakistan)

E-mail address: farooggujar@gmail.com 